\title{
Tensor products of ideal codes over Hopf algebras
}

\author{
J. Cuadra J.M. Garcia-Rubira J.A. Lopez-Ramos * \\ Departamento de Algebra y Análisis Matemático \\ Universidad de Almería \\ 04120 Almería, Spain
}

\begin{abstract}
We study indecomposable codes over the well-known family of Radford Hopf algebras. We use properties of Hopf algebras to show that tensors of ideal codes are ideal codes, extending the corresponding result given in [4] and showing that in this case, semisimplicity is lost.
\end{abstract}

Keywords: Radford Hopf algebra, ideal code, tensor product

\section{Introduction}

The more mathematical structure one can add to a system the more information and description can be obtained. A clear example of this occurs with algebraic codes. Linearity on a code implies, between other things that one has not to compute all distances between every pair of codewords. If the code is also cyclic, then we get fast encoding/decoding, etc. As Berman in [1] observed, cyclic codes and Reed-Muller codes can be seen as ideals in the group ring $\mathbb{K} G$ (where $\mathbb{K}$ is a finite field and $G$ is a finite cyclic group). This fact has led many authors to the study of codes from a point of view of Ring Theory (cf. [3] for instance). Recently Wood in [9] and [10] has stated the suitability of Frobenius rings when studing codes over a finite ring. More precisely he showed that a finite Frobenius ring is characterized by the fact of allowing the Extension Theorem for linear codes. One example of such rings are finite dimensional Hopf Algebras. In [4] the authors characterized all indecomposable codes over an important family of Hopf algebras. Codes in such a family of Hopf algebras are shown to be a concatenation of cyclic codes in $\mathbb{K}_{\mathbb{Z}_{n}}$. As an application of identifying the concatenation of ideal / codes with an ideal / code in a bigger algebra is that we can describe easily duals and tensors of such codes. To do so, dual and tensor product of ideal codes are equipped with an additional module structure given by the antipode and the comultiplication of the underlying Hopf algebra respectively. In [5] the authors considered a larger family of Hopf algebras, known as Radford Hopf algebras, that contains the first one. They give a

*corresponding author jlopez@ual.es 
decomposition theorem relating these two families of Hopf algebras and using it, duals of indecomposable ideal codes are characterized. These new results extend those in [4], showing that these codes are also concatenation of cyclic codes in a bigger algebra. Our aim in this paper is to study the behavior of tensors of indecomposable ideal codes in the Radford Hopf algebras. The second section is devoted to recall the main definitions of the Taft and Radford Hopf algebras. We give a sketch of the proof of the Theorem appearing in [5] that relates both families in order to understand the structure of the indecomposables in the family of Radford Hopf algebras. Following we start the study of tensors of indecomposables. We recall that in [4] we get that the tensor product of two any indecomposables in the Taft Hopf algebras is always a semisimple ideal code. Now we detach the fact that semisimplicity is lost when considering the family of Radford Hopf algebras (3.3). For prerequisites on Hopf algebras we refer the reader to [2] or [6].

\section{Two families of Hopf algebras}

Let $n>1$ be an integer and let $\mathbb{K}$ be a field containing a primitive $n$-th root of unity $\omega(\operatorname{char}(\mathbb{K}) \nmid n)$. The free algebra $\mathcal{H}=\mathbb{K}\langle g, x\rangle$ over the non-commutative indeterminates $g$ and $x$ is a bialgebra with comultiplication and counit given by $\Delta(g)=g \otimes g, \Delta(x)=x \otimes g+1 \otimes x$ and $\varepsilon(g)=1, \varepsilon(x)=0$ respectively. Let us consider the ideal $\Gamma$ of $\mathcal{H}$ generated by $g^{n}-1, x g-\omega g x$ and $x^{n}$. The quotient algebra, that we will denote by $T_{n}$, is a Hopf algebra (known as Taft Hopf algebra) with antipode given by $S(g)=g^{-1}$ and $S(x)=-x g^{-1}$. Observe that $\left\{g^{i} x^{j}: 0 \leq i, j,<n\right\}$ is a basis for $T_{n}$ and so $\operatorname{dim}\left(T_{n}\right)=n^{2}$. As noted before, indecomposable ideal codes over $T_{n}$ where studied in [4].

Now let $p>1$ be a prime number and assume $\operatorname{char}(\mathbb{K}) \neq p$. Let $\Gamma^{\prime}$ be the ideal of $\mathcal{H}$ generated by $g^{p n}-1, x g-\omega g x$, and $x^{n}-\left(g^{n}-1\right)$ and let $A_{p}=\mathcal{H} / \Gamma^{\prime}$. $A_{p}$ is also a Hopf algebra with antipode given by $S(g)=g^{-1}, S(x)=-x g^{-1}$ (as above, now $g$ and $x$ denote the corresponding projections of the original elements $g$ and $x$ in $\mathcal{H})$. We easily see that the set $B=\left\{g^{i} x^{j}: 0 \leq i<p n, 0 \leq j<n\right\}$ is a basis for $A_{p}$ and thus $\operatorname{dim}\left(A_{p}\right)=p n^{2}$.

This family of Hopf algebras was introduced by Radford in [7] as an example of Hopf algebra $H$ whose Jacobson radical $J(H)$ is not a Hopf ideal, i.e., the semisimple quotient algebra $H / J(H)$ does not admit a Hopf algebra structure making the canonical projection into a Hopf algebra map. We will refer to $A_{p}$ as Radford Hopf algebra.

The following result appearing in [5, Theorem 2.2] relates these the two families of Hopf algebras defined above. We include a sketch of the proof here in order to understand better the incoming results.

Theorem 2.1. Assume that $\mathbb{K}$ contains a primitive $p$-th root of unity $\theta$ and an $n$-th root of $\theta$. Then

$$
A_{p} \cong T_{n} \oplus M_{n}(\mathbb{K}) \oplus \stackrel{(p-1)}{\cdots} \oplus M_{n}(\mathbb{K})
$$


as algebras.

Proof. $e=\frac{1}{p} \sum_{k=0}^{p-1} g^{k n}$ is a central idempotent in $A_{p}$ and it can be shown that $A_{p} e \cong T_{n}$ as algebras via the isomorphism $g \rightarrow g e, x \rightarrow x e$.

Now $A_{p}(1-e) \cong M_{n}(\mathbb{K}) \oplus \stackrel{(p-1)}{\cdots} \oplus M_{n}(\mathbb{K})$. To show this we can show that the set $\left\{f_{i}\right\}_{i=1}^{p-1}$ is a complete set of orthogonal central idempotents in $A_{p}(1-e)$, where

$$
f_{i}=\frac{1}{p}\left(\sum_{j=0}^{p-1} \theta^{i j} g^{n j}\right)(1-e)
$$

for $i=1, \ldots, p-1$. Now considering the Pierce decomposition in $A_{p} e^{\prime}$ corresponding to the set $\left\{f_{i}: i=1, \ldots, p-1\right\}$, that is,

$$
A_{p}(1-e)=\oplus_{i=1}^{p-1}\left(A_{p}(1-e)\right) f_{i}=\oplus_{i=1}^{p-1} A_{p} f_{i}
$$

we show that $A_{p} f_{i} \cong M_{n}(\mathbb{K})$ as algebras for every $i=1, \ldots, p-1$ via the isomorphism

$$
\begin{aligned}
g f_{i} \longrightarrow M & =\lambda^{p-i} \operatorname{diag}\left(1, \omega^{-1}, \ldots, \omega^{-(n-1)}\right) \\
x f_{i} \longrightarrow N & =\left(\begin{array}{ccccc}
0 & 1 & 0 & \cdots & 0 \\
0 & 0 & 1 & \cdots & 0 \\
\vdots & \vdots & \vdots & & \vdots \\
0 & 0 & 0 & \cdots & 1 \\
\theta^{p-i}-1 & 0 & 0 & \cdots & 0
\end{array}\right)
\end{aligned}
$$

and where $\lambda \in \mathbb{K}$ is the $n$-th root of $\theta$ given by hypothesis.

\section{Tensor Product of indecomposables}

Our aim is to study the tensor product of indecomposables in $A_{p}$. Thus, by the precedent Theorem we have to consider, on one hand, those in $T_{n}$, and on the other, those corresponding to the matrix blocks. Indecomposable codes in $T_{n}$ are discussed in [4] and we will refer to them as indecomposable Taft codes. Through the next subsection we recall their structure.

\subsection{Indecomposables Taft codes}

Recall that $T_{n}=\mathbb{K}\left\langle g, x: g^{n}=1, x^{n}=0, x g=\omega g x\right\rangle, \operatorname{dim}\left(T_{n}\right)=n^{2}$ and a basis for $T_{n}$ is $B=\left\{g^{i} x^{j}: 0 \leq i, j,<n\right\}$. The Jacobson radical $J\left(T_{n}\right)$ of $T_{n}$ coincides with the ideal generated by $x$. Then the semisimple quotient algebra $\bar{T}_{n}=T_{n} / J\left(T_{n}\right)$ is isomorphic to the group algebra $\mathbb{K Z}_{n}$ via the map sending $g \mapsto g$ and $x \mapsto 0$. The primitive idempotents of $\mathbb{K} \mathbb{Z}_{n}$,

$$
e_{l}=\frac{1}{n}\left(\sum_{i=0}^{n-1} \omega^{l i} g^{i}\right), \quad 0 \leq l<n,
$$


will give us the complete set $\left\{S_{l}: 0 \leq l<n\right\}$ of isomorphism classes of simple $T_{n}$-modules. Writing $u_{l}=e_{l}$ and $S_{l}=\bar{T}_{n} e_{l}=\mathbb{K} u_{l}$ we get that $g \cdot u_{l}=\omega^{-l} u_{l}$ and $x \cdot u_{l}=0$. By lifting this set of orthogonal primitive idempotents to one of $T_{n}$ we will obtain the set of isomorphism classes of projective indecomposable modules. Each of them is represented by the projective cover $P_{l}$ of $S_{l}$. Observe that $e_{l}$, viewed as an element in $T_{n}$, provides a desired lifted idempotent of $e_{l}$. Therefore the above set is indeed a complete set of primitive orthogonal idempotents in $T_{n}$. Then $P_{l}=H e_{l}$ is the projective cover of the simple $\bar{T}_{n} e_{l}$. Setting $v_{i}=x^{i} e_{l}$ for $i=0, \ldots, n-1$, the set $\left\{v_{0}, \ldots, v_{n-1}\right\}$ is a basis of $P_{l}$. The action of $T_{n}$ on these elements is given by:

$$
\begin{aligned}
g \cdot v_{i} & =\omega^{-(l+i)} v_{i} \\
x \cdot v_{i} & =v_{i+1}, \quad i=0, \cdots, n-2, \\
x \cdot v_{n-1} & =0
\end{aligned}
$$

As $T_{n}$ is a serial algebra, the only submodules of $P_{l}$ are $N_{l, j}=J\left(T_{n}\right)^{j} P_{l}$ for $j=0, \ldots, n$. Taking into account $(1)$, a basis for $N_{l, j}$ is $\left\{v_{j}, \ldots, v_{n-1}\right\}$ and so $\operatorname{dim}\left(N_{l, j}\right)=n-j$. Then

$$
\{0\}=N_{l, n} \subset N_{l, n-1} \subset \cdots \subset N_{l, 2} \subset N_{l, 1} \subset N_{l, 0}=P_{l}
$$

is a composition series of $P_{l}$ and the composition factors are $N_{l, k} / N_{l, k+1} \cong S_{l+k}$ for $k=0, \ldots, n-1$. We claim that the quotient module $P_{l} / N_{l, j}$ is isomorphic to $N_{l+j, n-j}$. For, denote by $[v]$ the class of $v \in P_{l}$. Then $\left\{\left[v_{0}\right], \ldots,\left[v_{j-1}\right]\right\}$ is a basis of $P_{l} / N_{l, j}$. The module structure is given by:

$$
\begin{aligned}
g \cdot\left[v_{i}\right] & =\left[g \cdot v_{i}\right] \\
& =\left[\omega^{-(i+l)} v_{i}\right] \\
& =\omega^{-(i+l)}\left[v_{i}\right], \quad i=0, \ldots, j-1, \\
x \cdot\left[v_{i}\right] & =\left[x \cdot v_{i}\right] \\
& =\left[v_{i+1}\right], \quad i=0, \ldots, j-2, \\
x \cdot\left[v_{j-1}\right] & =\left[x \cdot v_{j-1}\right] \\
& =\left[v_{j}\right] \\
& =[0]
\end{aligned}
$$

Hence the map $N_{l+j, n-j} \rightarrow P_{l} / N_{l, j}, v_{n-i} \mapsto\left[v_{j-i}\right]$ for $i=1, \ldots, j$ is an isomorphism of modules.

For a characterization of the tensor product of two any indecomposables Taft codes we refer the reader to [4].

\subsection{Product of two simples in the matrix part}

We recall that any matrix block $M_{n}(\mathbb{K})$ is generated by a simple $S_{j}$, i.e., $M_{n}(\mathbb{K}) \cong S_{j}^{(n)}$ for $j=1, \ldots, p-1$. By the proof of Theorem 2.1 it follows that the action of the generators of $A_{p}$ over the basis of these simples is as follows: given $S_{j}=\mathbb{K}\left\{v_{0}, \ldots v_{n}\right\}(j=1, \ldots, p-1)$ 


$$
\begin{array}{ll}
g \cdot v_{i} & =\lambda^{(p-j)} \omega^{-i} v_{i} \\
x \cdot v_{i} & =v_{i+1}, \quad i=0, \ldots, n-2 \\
x \cdot v_{n-1} & =\left(\theta^{(p-j)}-1\right) v_{0}
\end{array}
$$

Lemma 3.1. $S_{j} \otimes S_{j} \cong S_{k}^{(n)}$ for $j=1, \ldots p-1$ and $k \equiv_{p} 2 j$ as $A_{p}$-modules.

Proof. Let us write $S_{j} \otimes S_{j}=\mathbb{K}\left\{z_{r, s}: 0 \leq r, s \leq n-1\right\}$ for $z_{r, s}=v_{r} \otimes v_{s}$. Let us show the action of $g$ on $z_{r, s}$.

$$
\begin{aligned}
g z_{r, s} & =g v_{r} \otimes g v_{s}=\omega^{-r} \lambda^{p-j} v_{r} \otimes \omega^{-s} \lambda^{p-j} v_{s}=\omega^{-(r+s)} \lambda^{2(p-j)} v_{r} \otimes v_{s}= \\
& =\omega^{-(r+s)} \lambda^{2(p-j)} z_{r, s}
\end{aligned}
$$

So let us consider $z_{k, l}$ such that $k+l \equiv_{n} 0$ and let $t_{i}=x^{i} z_{k, l}$ for $i=0, \ldots, n-$ 1 and let us calculate the action of $x$ and $g$ on the elements of $B=\left\{t_{0}, \ldots, t_{n-1}\right\}$ :

$$
\begin{aligned}
& \qquad \begin{array}{l}
g t_{i}=g\left(x^{i} z_{k, l}\right)=\omega^{-i} x^{i}\left(g z_{k, l}\right)=\omega^{-i} x^{i}\left(\omega^{-(k+l)} \lambda^{2(p-j)} z_{k, l}\right)=\omega^{-i} \lambda^{2(p-j)} x^{i} z_{k, l} \\
=\omega^{-i} \lambda^{2(p-j)} t_{i},
\end{array} \\
& \text { for } i=0, \ldots, n-1
\end{aligned}
$$

since $\lambda^{n}=\theta$. Thus if $z_{k, l}$ is such that $k+l \equiv_{n} 0$ we get, on one side, by the action of $g$ on the elements of $B$, that $B$ is a basis since every $t_{i} i=0, \ldots, n-1$ is an eigenvector associated to a different eigenvalue and, on the other hand that $\mathbb{K}\left\{t_{0}, t_{1}, \ldots, t_{n-1}\right\} \cong S_{2 j}$ as $A_{p}$-modules. By a reasoning on the dimension of the vector spaces we get that $S_{j} \otimes S_{j} \cong S_{k}^{(n)}, k=2 j(\bmod p)$ as $A_{p}$-modules.

Lemma 3.2. $S_{j} \otimes S_{k} \cong S_{j+k}^{(n)}$ for $j+k<p$ and $j \neq k$ as $A_{p}$-modules.

Proof. Analogous to Lemma 3.1.

Lemma 3.3. $S_{j} \otimes S_{k} \cong \oplus_{l=0}^{n-1} P_{l}$ for $j+k=p$ as $A_{p}$-modules, where $P_{l}$ denotes the indecomposable projective ideal code corresponding to the simple ideal code $S_{l}$ in $T_{n}$.

Proof. As in the previous results let us write $S_{j} \otimes S_{k}=\mathbb{K}\left\{z_{r, s}: 0 \leq r, s \leq n-1\right\}$ for $z_{r, s}=v_{r} \otimes v_{s}$ and let us show the action of $g$ on $z_{r, s}$.

$$
g z_{r, s}=g \omega^{-(r+s)} \lambda^{(p-j+p-k)} z_{r, s}=\omega^{-(r+s)} \lambda^{p} z_{r, s}=\omega^{-(r+s+l)} z_{r, s}
$$

for some $l$ since $1=\theta^{p}=\left(\lambda^{n}\right)^{p}=\left(\lambda^{p}\right)^{n}$ and thus $\lambda^{p}=\omega^{-l}$ for some $l$.

Let us consider now $z_{r, 0}$ for $r=0, \ldots, n-1$. Then $g z_{r, 0}=\omega^{-(r+l)} z_{r, 0}$ for every $r=0, \ldots, n-1$. Let us define $t_{i, r}=x^{i} z_{r, 0} i=0, \ldots, n-1$. The action of $g$ and $x$ on these elements is 


$$
\begin{gathered}
g t_{i, r}=g\left(x^{i} z_{r, 0}\right)=\omega^{-i} x^{i} g z_{r, 0}=\omega^{-i} x^{i} \omega^{-(r+l)} z_{r, 0}=\omega^{-(i+r+l)} t_{i, r} \\
x t_{i, r}=x\left(x^{i} z_{r, 0}\right)=x^{i+1} z_{r, 0} t_{i+1, r} \quad \text { for } r=0, \ldots, n-2 \\
x t_{n-1, r}=x^{n} z_{r, 0}\left(g^{n}-1\right) z_{r, 0}=\left(\omega^{-(i+r+l) n}-1\right) z_{r, 0}=0
\end{gathered}
$$

As in Lemma 3.1 we get that $\left\{t_{0, r}, t_{1, r}, \ldots, t_{n-1, r}\right\}$ is a linear independent set of vectors and from the definition of $P_{r+l}$ we get that $\mathbb{K}\left\{t_{0, r}, t_{1, r}, \ldots, t_{n-1, r}\right\} \cong$ $P_{r+l}$. Again, by an argument on the dimensions we get that

$$
S_{j} \otimes S_{k} \cong \oplus_{l=0}^{n-1} P_{l+r} \cong \oplus_{l=0}^{n-1} P_{l}
$$

Lemma 3.4. $S_{j} \otimes S_{k} \cong S_{q}^{(n)}$ for $j+k=p+q$ as $A_{p}$-modules.

Proof. Let $q$ be such that $j+k=p+q$ and let $z_{r, s}$ the same elements considered in Lemma 3.3. Then

$$
\begin{aligned}
g z_{r, s} & =g v_{r} \otimes g w_{s}=\omega^{-r} \lambda^{p-j} v_{r} \otimes \omega^{-s} \lambda^{p-k} w_{s}=\omega^{-(r+s)} \lambda^{p-j+p-k} v_{r} \otimes v_{s}= \\
& =\omega^{-(r+s)} \lambda^{p-j+p-k} z_{r, s}=\omega^{-(r+s)} \lambda^{p-j-k} \lambda^{p} z_{r, s} \\
& =\omega^{-(r+s)} \lambda^{-q} \omega^{-l} z_{r, s}
\end{aligned}
$$

for some $l$, as in the previous result. Now let $t_{0, h}=x^{h} z_{r, s}$ such that $h+l \equiv_{n} 0$ and $r+s \equiv_{n} 0$. Thus we get that

$g t_{0, h}=g x^{h} z_{r, s}=\omega^{-h} x^{h} g z_{r, s}=\omega^{-h} x^{h} \omega^{-(r+s)} \lambda^{-q} \omega^{-l} z_{r, s}=\omega^{-(h+l)} \lambda^{-q} x^{h} z_{r, s}=$ $\lambda^{-q} t_{0, h}$

Consider now the elements $t_{i, h}=x^{i} t_{0, h}, i=0, \ldots, n-1$ and as before, let us show the actions of $g$ and $x$ on these.

$$
\begin{gathered}
g t_{i, h}=g x^{i} t_{0, h}=\omega^{-i} x^{i} g t_{0, h}=\omega^{-i} x^{i} \lambda^{-q} t_{0, h}=\omega^{-i} \lambda^{-q} x^{i} t_{0, h}=\omega^{-i} \lambda^{-q} t_{i, h} \\
x t_{i, h}=x\left(x^{i} t_{0, h}=x^{i+1} t_{0, h}=t_{i+1, h}, \quad i=0, \ldots, n-2\right. \\
x t_{n-1, h}=x\left(x^{n-1} t_{0, h}\right)=x^{n} t_{0, h}=\left(g^{n}-1\right) t_{0, h}=\left(\lambda^{-q n}-1\right) t_{0, h}=\left(\lambda^{p-q}-1\right) t_{0, h}
\end{gathered}
$$

Therefore we get that $\mathbb{K}\left\{t_{0, h}, x t_{0, h}, \ldots, x^{n-1} t_{0, h}\right\} \cong S_{q}$ and by a dimension argument $S_{j} \otimes S_{k} \cong S_{q}^{(n)}$.

By the precedent Lemmas we get the following:

Theorem 3.5. The tensor product of two any simple ideal codes in the matrix part of the decomposition of $A_{p}$ is either a (finite) direct sum of copies of a simple ideal code in the matrix part or a (finite) direct sum of projective ideal codes in the Taft algebra. 


\subsection{Product of an indecomposable Taft code with a simple in the matrix part}

Following the notation of Subsection 3.1 we will denote indecomposables Taft codes by $N_{r, s}$. The following Theorem characterizes the structure of a tensor product of such a indecomposable Taft code and a simple code in the matrix part of the decomposition of $A_{p}$, say $S_{j}$.

Theorem 3.6. $N_{r, s} \otimes S_{j} \cong S_{j}^{(n-s)} \cong S_{j} \otimes N_{r, s}$ for every $j=1, \ldots, p-1$ and every $r, s=0, \ldots, n-1$.

Proof. Let $z_{k, l}=u_{k} \otimes v_{l} n-s \leq k \leq n-1$ and $0 \leq l \leq n-1$ an element of a basis of $N_{r, s} \otimes S_{j}$. Then the actions of $g$ and $x$ on these elements are

$$
\begin{aligned}
& g z_{k, l}=g u_{k} \otimes g v_{l}=\omega^{-(r+k)} u_{k} \otimes \omega^{-l} \lambda^{p-j} v_{l}=\omega^{-(r+k+l)} \lambda^{p-j} u_{k} \otimes v_{l} \\
& =\omega^{-(r+k+l)} \lambda^{p-j} z_{k, l}
\end{aligned}
$$

for $n-s \leq k \leq n-1$ and $0 \leq l \leq n-1$.

$x z_{k, l}=1 \cdot u_{k} \otimes x v_{l}+x u_{k} \otimes g v_{l}=u_{k} \otimes v_{l+1}+u_{k+1} \otimes \omega^{-l} \lambda^{p-j} v_{l}=z_{k, l+1}+\omega^{-l} \lambda^{p-j} z_{k+1, l}$

for $n-s \leq k \leq n-2$ and $0 \leq l \leq n-2$.

$x z_{n-1, l}=1 \cdot u_{n-1} \otimes x v_{l}+x u_{n-1} \otimes g v_{l}=u_{n-1} \otimes v_{l+1}+0 \otimes \omega^{-l} \lambda^{p-j} v_{l}=z_{n-1, l+1}$

$l=0, \ldots, n-2$

$x z_{n-1, n-1}=1 \cdot u_{n-1} \otimes x v_{n-1}+x u_{n-1} \otimes g v_{n-1}=u_{n-1} \otimes\left(\theta^{p-j}-1\right) v_{0}+0 \otimes \omega^{-l} \lambda^{p-j} v_{n-1}$

$=z_{n-1,0}$

Since $\omega$ is an $n$-th primitive root of unit we get that $\omega^{-(r+k+l)}=1$ if and only if $r+k+l \equiv_{n} 0$. Now for fixed $r, r=0, \ldots, n-1$, the equation $k+l \equiv_{n} n-r$ has a different solution for each $k, k=n-s, \ldots, n-1$.

Let us consider now $z_{k, l}$ such that $r+k+l \equiv_{n} 0$ and define $t_{i}=x^{i} z_{k, l}$. Then we get that

$$
\begin{aligned}
& g t_{i}=g\left(x^{i} z_{k, l}\right)=\omega^{-i} x^{i} g z_{k, l}=\omega^{-i} x^{i} \omega^{-(k+r+l)} \lambda^{p-j} z_{k, l}=\omega^{-i} x^{i} \lambda^{p-j} z_{k, l}= \\
& =\omega^{-i} \lambda^{p-j} t_{i}
\end{aligned}
$$

From this we get, as in previous reasonings, that $\left\{t_{i}: i=0, \ldots, n-1\right\}$ is a basis.

The action of $x$ on the elements of this basis is as follows: 


$$
x t_{i}=x x^{i} z_{k, l}=x^{i+1} z_{k, l}=t_{i+1} \text { for } i=0, \ldots, n-2
$$

$x t_{n-1}=x\left(x^{n-1} z_{k, l}\right)=x^{n} z_{k, l}=\left(g^{n}-1\right) z_{k, l}=\left(g^{n}-1\right) t_{0}=\omega^{-(k+r+l)}\left(\lambda^{p-j}\right)^{n} t_{0}-t_{0}$ $=\left(\theta^{p-j}-1\right) t_{0}$ since $\lambda^{n}=\theta$ and $\omega^{n}=1$.

In this way, for each of the $s$ possible elections of $z_{k, l}$ we get that

$$
\mathbb{K}\left\{z_{k, l}, x z_{k, l}, \ldots, x^{n-1} z_{k, l}\right\} \cong S_{j}
$$

Thus $N_{r, s} \otimes S_{j} \cong S_{j}^{(n-s)}$.

An analogous reasoning gives that $S_{j} \otimes N_{r, s} \cong S_{j}^{(n-s)}$.

\section{Some practical considerations}

We recall from [4, Section 5] that indecomposable projective codes in the Taft Hopf algebra (those appearing in 3.3) are a concatenation of cyclic codes and a generator matrix of the indecomposable projective $P_{l}(l=0, \cdots, n-1)$ is

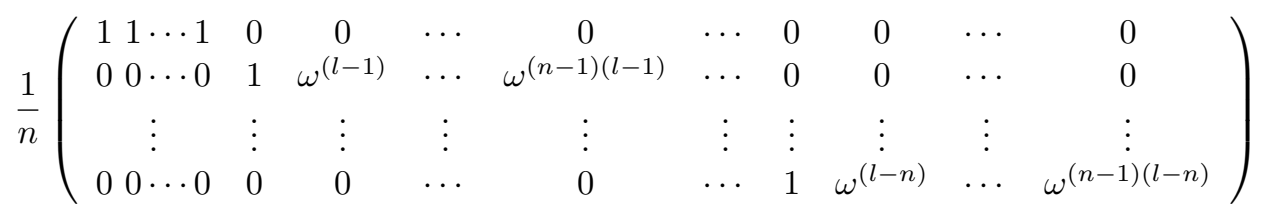

for $\omega$ being a $n$-th root of unity.

Simple codes in the matrix part may be also described easily and this is given in [5, Section IV]. A generator matrix for a simple code $S_{j}$ in the matrix part is given, as above, by a block matrix,

$$
N_{j}=\frac{1}{p n}\left(\begin{array}{cccccc}
B_{0} & \overline{0} & \cdots & \overline{0} & \cdots & \overline{0} \\
\overline{0} & B_{1} & \cdots & \overline{0} & \cdots & \overline{0} \\
\vdots & \vdots & \vdots & \vdots & \vdots & \vdots \\
\overline{0} & \overline{0} & \cdots & \overline{0} & \cdots & B_{n-1}
\end{array}\right)
$$

where $\overline{0}$ denotes a block of $p n$ zeros and the block $B_{m}, m=0, \ldots, n-1$ of length $p n$, is placed in position $m$. This block is given by $B_{m}=\left(B_{m}, \ldots, B_{m p-1}\right)$ with each $B_{m} l$ being the block of length $n$ given by

$$
B_{m l}=\theta^{l j}\left(1, \lambda^{p-j}, \lambda^{2(p-j)}, \ldots, \lambda^{(n-1)(p-j)}\right)
$$

When considering linear codes, the tensor product of two linear codes can be viewed as a concatenation of these two linear codes (we are considering the usual linear structure) and, thus, the minimum distance of this new code is the minimum of the corresponding two minimum distances. What we have 
considered through this paper is the tensor product of two ideal codes and got a structure as ideal code on this new code induced by the comultiplication of the considered Hopf algebra. Now we have got that this structure of ideal code can be characterized depending on the case and, from Lemmas 3.1, 3.2 and 3.4 we get codes that do not include the codes involved in the tensor product. Codes obtained in these Lemmas are concatenation of copies of one determined code in each case. The first difference with the linear case is that the dimension is not the sum of the codes. Secondly, by Lemmas 3.1, 3.2 and 3.4 and from the above matrix $N_{j}$, we get that the tensor codes obtained in these Lemmas have minimum distance $p n$, analogously to the case of linear codes. However, Lemma 3.3 offers interesting consequences from the Coding Theory and Ring Theory points of view respectively. From the Coding Theory point of view we have to remark that dimensions behaves as in Lemmas 3.1, 3.2 and 3.4, but the minimum distance decreases. From Lemma 3.3 and the matrix $M_{l}$ we get that the minimum distance of the corresponding tensor product is $n$, being the minimum distance of the original codes appearing in the tensor product equal to $p n$. From the Ring Theory point of view we get that the tensor product of two ideal codes as in Lemma 3.3 is not semisimple.

Lastly, but not less important, we can observe that tensor products of indecomposables codes in the Radford algebra is always a concatenation of cyclic codes cf. [4] and [5]. Thus, although they do not have a great behavior concerning the minimum distance, they are of easy and fast implementation by blocks and suitable in situations where a limited number of errors can appear in every block, such as the so-called byte error correcting codes (cf. [8] for examples).

\section{Acknowledgements}

This work was partially supported by projects MTM2008-03339 from MICINN, P07-FQM03128 from Junta de Andalucía and TEC2009-13763-C02-02.

\section{References}

[1] Berman J. On the Theory of Group Codes. Kibernetika (Kiev) 1967, 1:31-39 (Russian): translated as Cybernetics, 1969, 1: 25-39.

[2] Dăscălescu S, Năstăsescu C,Raianu S. Hopf Algebras. An Introduction, Marcel Dekker, Inc., 2001.

[3] Greferath M, Nechaev A, Wisbauer R. Finite Quasi-Frobenius Modules and Linear Codes. J Algebra Appl, 2004, 3 (3): 247-272.

[4] Cuadra J, García-Rubira J M, López-Ramos J A. Determining all indecomposable codes over some Hopf algebras. J Comput Appl Math 2011, 235 (7): 1833-1839. 
[5] Cuadra J, García-Rubira J M, López-Ramos J A. Codes as Ideals Over Some Pointed Hopf Algebras, Proceedings of the 19th International Symposium on Mathematical Theory of Networks and Systems - MTNS 2010, Budapest, July 2010, 1287-1291.

[6] Montgomery S. Hopf Algebras and Their Actions on Rings, CBMS 82 A M S, 1982.

[7] Radford D E. On the coradical of a finite-dimensional Hopf algebra. Proc Amer Math Soc, 1975, 53 (1): 9-15.

[8] Rao T R N, Fujiwara E. Error-Control Coding for Computer Systems, Prentice Hall Inc, 1989.

[9] Wood J A. Duality for modules over finite rings and applications to coding theory. Amer J Math, 1999, 121 (3): 555-575.

[10] Wood J A. Code equivalence characterizes finite Frobenius rings. Proc Amer Math Soc, 2008, 136 (2): 699-706 (electronic). 